\title{
The role of lateral magnetic reconnection in solar eruptive events
}

\author{
A. Soenen ${ }^{1,2}$, A. Bemporad ${ }^{3}$, C. Jacobs ${ }^{1,2}$, and S. Poedts ${ }^{1,2}$ \\ ${ }^{1}$ Centre for Plasma-Astrophysics, K.U. Leuven, Belgium \\ ${ }^{2}$ Leuven Mathematical Modeling \& Computational Science Research Center, Belgium \\ ${ }^{3}$ INAF - Torino Astrophysical Observatory, via Osservatorio 20, 10025 Pino Torinese (TO), Italy
}

Received: 29 May 2009 - Revised: 25 September 2009 - Accepted: 6 October 2009 - Published: 16 October 2009

\begin{abstract}
On 10-11 December 2005 a slow CME occurred in between two coronal streamers in the Western Hemisphere. SOHO/MDI magnetograms show a multipolar magnetic configuration at the photosphere consisting of a complex of active regions located at the CME source and two bipoles at the base of the lateral coronal streamers. White light observations reveal that the expanding CME affects both of the lateral streamers and induces the release of plasma within or close to them. These transient phenomena are possibly due to magnetic reconnections induced by the CME expansion that occurs either inside the streamer current sheet or between the CME flanks and the streamer. Our observations show that CMEs can be associated to not only a single reconnection process at a single location in the corona, but also to many reconnection processes occurring at different times and locations around the flux rope. Numerical simulations are used to demonstrate that the observed lateral reconnections can be reproduced. The observed secondary reconnections associated to CMEs may facilitate the CME release by globally decreasing the magnetic tension of the corona. Future CME models should therefore take into account the lateral reconnection effect.
\end{abstract}

Keywords. Solar physics, astrophysics, and astronomy (Corona and transition region; Flares and mass ejections) Space plasma physics (Magnetic reconnection)

\section{Introduction}

Coronal Mass Ejections (CMEs) usually refer to spectacular eruptions of plasma and embedded coronal magnetic field from the Sun's corona into interplanetary space. The total mass ejected in CMEs ranges from $10^{13} \mathrm{~g}$ to a few $10^{16} \mathrm{~g}$,

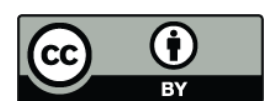

Correspondence to: A. Soenen (alexander.soenen@wis.kuleuven.be) the total energy from $10^{27}$ erg to some $10^{33} \mathrm{erg}$ (see e.g. Vourlidas et al., 2002; Gopalswamy et al., 2004). Based on thousands of CMEs observed by Solwind, SMM (the "Solar Maximum Mission"), and SOHO (the "Solar and Heliospheric Observatory"), several statistical properties of larger solar eruptions have been studied, such as velocity, acceleration, and angular width distributions. Some candidates for the CME triggering, suggested from observational data analysis, are: the proximity of a CME site to coronal holes (Bravo et al., 1999), magnetic shear (Mikic and Linker, 1994), filament helicity (Martin, 2003), X-ray sigmoids (Rust and Kumar, 1996; Sterling and Hudson, 1997) and magnetic flux emergence (Feynman and Martin, 1995).

In agreement with these different candidates, various CME models have been formulated invoking different scenarios for the storage and release of magnetic energy eventually leading to CMEs. A few excellent papers are available in literature that give a thorough review on the different CME initiation theories and models (Klimchuk, 2001; Low, 2001; Forbes et al., 2006; Roussev and Sokolov, 2006; Mikic and Lee, 2006). A large portion of the CME initiation models are considered to be storage and release models (Klimchuk, 2001). In these models, additional free magnetic energy can be stored in the corona due to photospheric surface flows or the emergence and cancellation of magnetic flux. The role of photospheric motions has been investigated numerically (see for example, van Ballegooijen and Martens, 1989; Amari et al., 2003), where the potential magnetic field is subjected to twisting motions so that a more complex structure is reached. Photospheric shearing motions have been the principal triggering mechanism used in the breakout scenario (Antiochos et al., 1999), where the vulnerability of multipolar topologies to rearrangements of the magnetic field's connectivity is exploited to enable the eruption. The sheared arcade reconnects with the overlying field to create a passage for the CME. More complex, three-dimensional (3-D) simulations were recently performed by Lynch et al. (2008). An alternative model uses

Published by Copernicus Publications on behalf of the European Geosciences Union. 

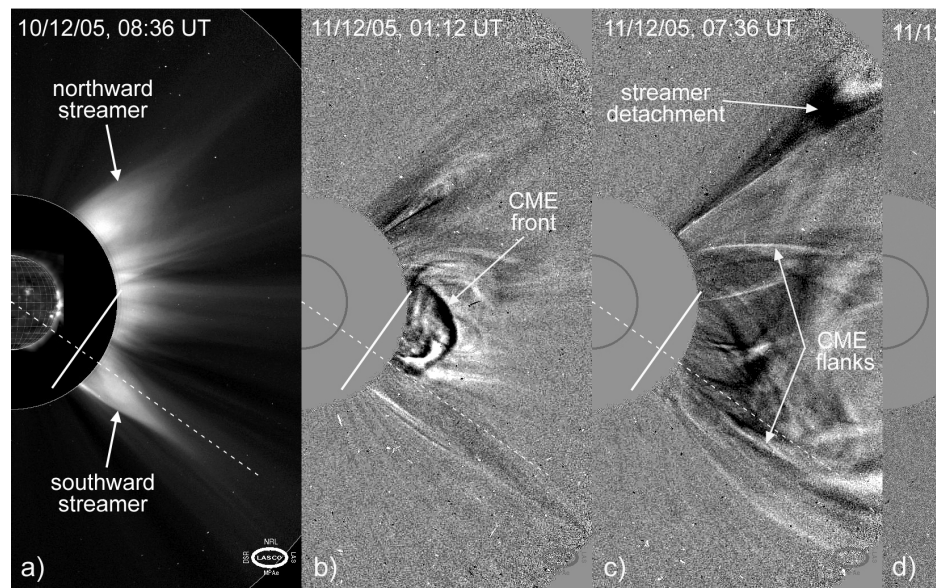

11112:05, $13 \cdot 12$ UT:

$11 / 12 / 05,21: 12,1 \%$

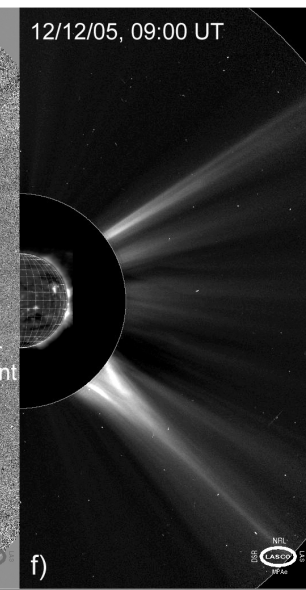

a)
b).

c)
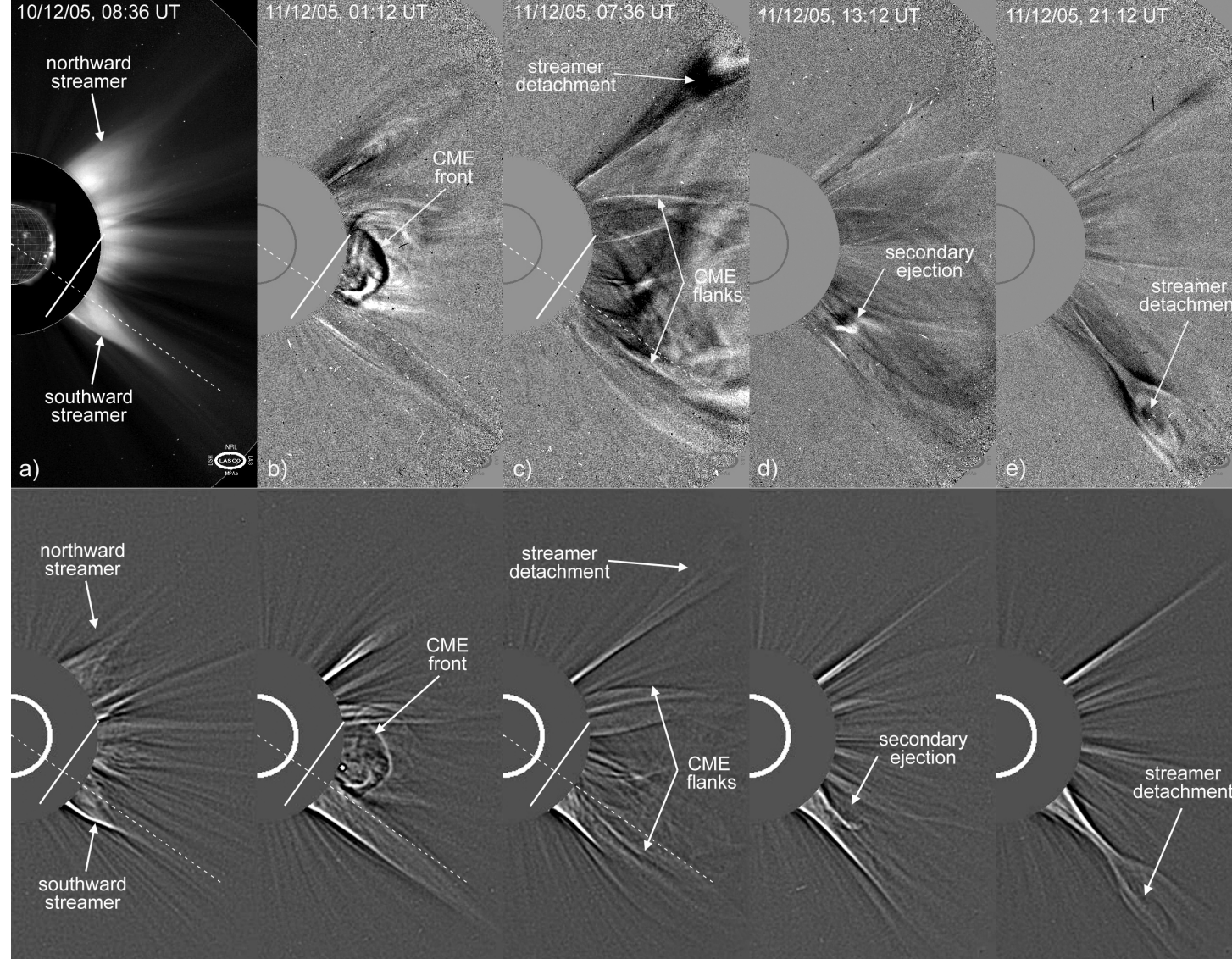

streamer

$\longrightarrow$
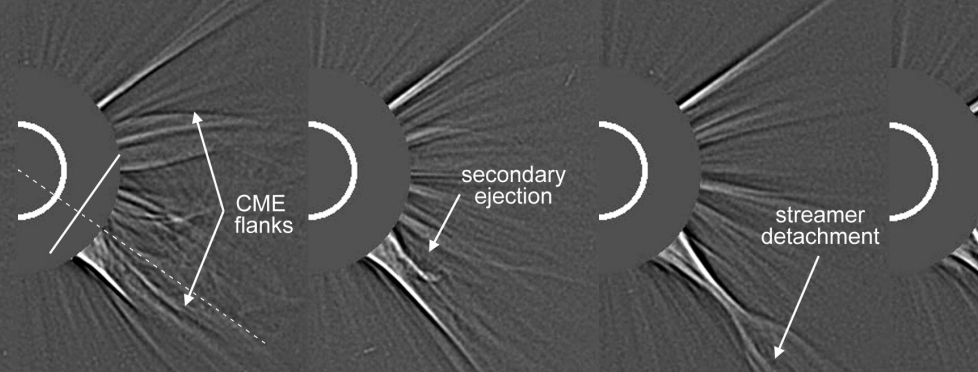

Fig. 1. The white light solar corona as seen by the LASCO/C2 coronagraph in standard (top row, panels a and f), running difference (panels b-e) and wavelet (bottom row) images. This sequence shows the observed occurrence of secondary eruptions associated with the main CME of 11 December 2005.

flux injection, or flux emergence, as described by Chen and Shibata (2000). In this model, a pre-existing flux rope is made unstable by the emergence of new magnetic flux of opposite polarity in the pre-existing coronal loops, causing a decrease in magnetic pressure that eventually leads to the formation of a current sheet. Recently, Zuccarello et al. (2008) showed that the emergence of new magnetic flux of the same sign of the central arcade of a breakout configuration can cause CMEs. In flux cancellation models, the magnetic field is first energized by shearing motions followed by converging motions towards the polarity inversion line. This process caused magnetic reconnection to occur between the opposite polarity feet of the sheared magnetic arcade. This eventually leads to the formation of a flux rope. Amari et al. (2000) investigated, in a simple bipolar topology, the role of flux cancellation in the destabilization of a flux rope formed by the combination of shearing and twisting motion and later studied this in a complex multiflux configuration (Amari et al., 2007).
However, the essential ingredients for CME onset are not yet identified and despite the huge amount of coronagraphic and spectroscopic data acquired in the last decades and the complexity reached by the most recent MHD simulations, the fundamental and still unresolved question about CMEs is: what really causes a CME to erupt?

Observations of large scale CMEs are not the whole story: over the last few years many small scale transient events and small scale eruptions have also been observed such as "narrow CMEs" (Gilbert et al., 2001), plasma blobs propagating outwards along post-CME and streamer current sheets (Ko et al., 2003), small scale recursive eruptions within the streamer boundaries namely "streamer puffs" (see Bemporad et al., 2005). More recent observations acquired from the Hinode and STEREO (the "Solar Terrestrial Relations Observatory") missions are now demonstrating that eruptions on the Sun are occurring on much smaller spatial scales and much higher rate than ever thought. In particular, Hinode/XRT and SOT data revealed that X-ray jets on polar coronal holes are much more frequent than previously detected from Yohkoh data 

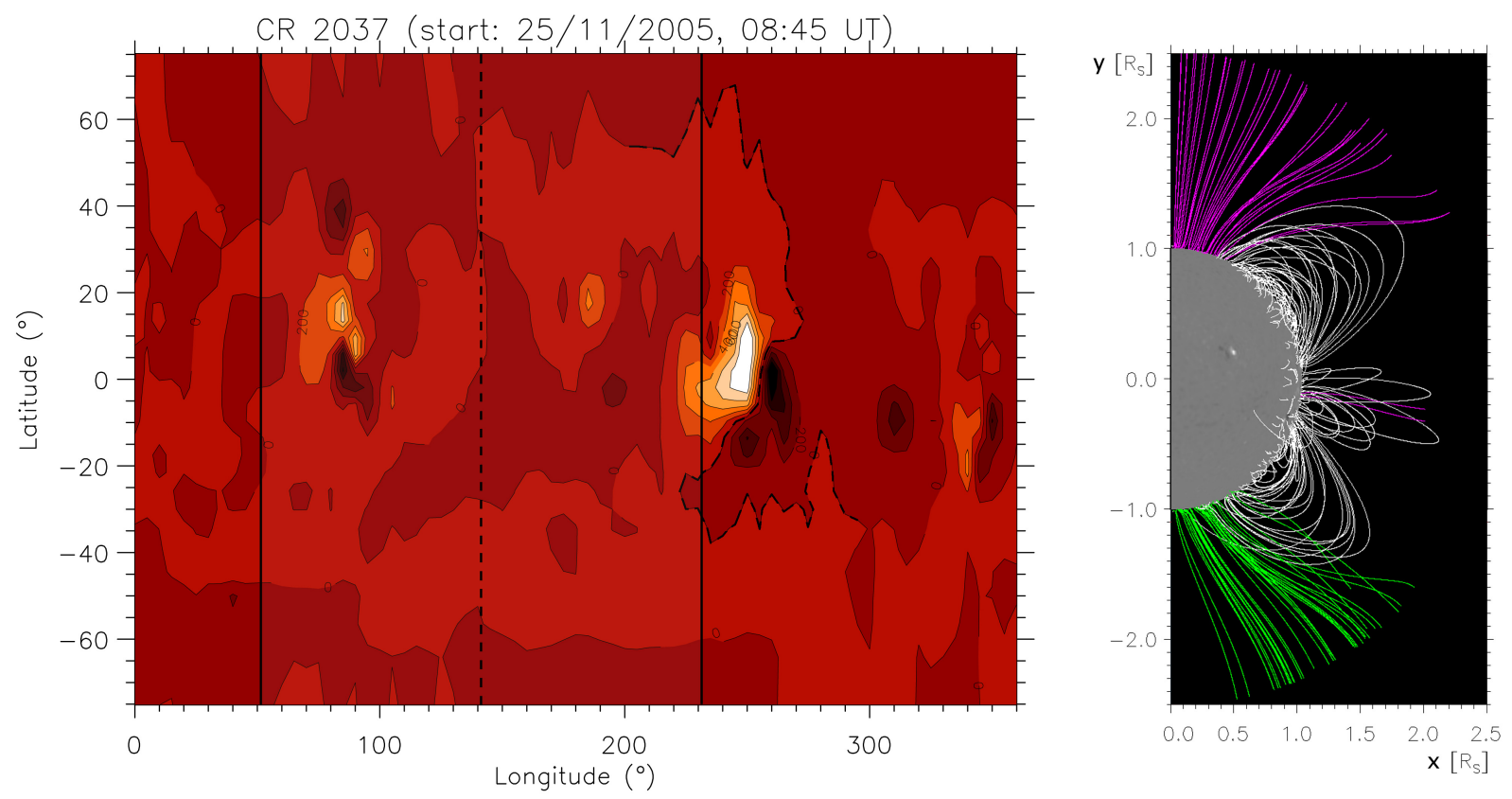

Fig. 2. Left: A map of photospheric magnetic fields observed during the transit of the AR complex across the solar disk (Carrington Rotation 2037); colors range from $-10^{3} \mathrm{G}$ (black) up to $+10^{3} \mathrm{G}$ (white). This map has been obtained by averaging the original magnetic field measurements by SOHO/MDI instrument, in order to reduce the complexity around the CME source region. Vertical solid lines delimit the solar hemisphere visible on 11 December 2005, while the dashed curved line shows the position of the neutral line across the West solar limb. Right: the pre-eruption coronal magnetic field configuration as extrapolated (with a Potential Field Source Surface approximation) above the West limb.

(Cirtain et al., 2007) and that small jets, similar to the Xray anemone jets, are occurring even above active regions in the chromosphere (Shibata, 2007). Very recently, Innes et al. (2009) demonstrated from high resolution $\left(\sim 1.6^{\prime \prime} /\right.$ pixel $)$ STEREO/EUVI data that mini-filament eruptions and microflares brightening occur at a rate of 1400 events/day over the whole Sun involving small spatial scales $\left(\sim 10^{\prime \prime}-30^{\prime \prime}\right)$, while small-scale transient events with typical three part structure - "mini CMEs" - from inside a coronal hole have been reported with STEREO/SECCHI data by Nisticò et al. (2009). Moreover, many previous works demonstrated that large and small scale eruptive events are quite often interrelated each other, possibly by cause-effect relationships (see van DrielGesztelyi et al., 2008, for a review on this subject): during their expansion a CME may reconnect with surrounding smaller-scale bipoles, leading to the observed large scale EUV dimmings (see Attrill et al., 2007; Mandrini et al., 2007), but also with fieldlines left open by a previous CME and/or with nearby streamers, generating type III and $\mathrm{N}$ radio burst (see e.g. Goff et al., 2007; Démoulin et al., 2007). There is also mounting evidence that coronal streamers can produce by themselves small scale eruptions: as it has been recently demonstrated, these structures are intrinsically unstable above their cusps, where reconnections may occur (Chen et al., 2009) and one or more plasmoids may form (Bárta et al., 2008), as also anticipated by the detection of propagating blobs, "in \& out pairs", and "streamer detachments" (see e.g. Wang and Sheeley, 2006; Sheeley and Wang, 2007); but coronal streamers are also known to be unstable below their cusp, where compact ejective flares occurring in the outer flank of the streamer base can produce many homologous small scale eruptions traveling out along the streamer and leaving it largely intact (Bemporad et al., 2005; Moore and Sterling, 2007).

All these results led us to consider the possibility that small scale, high rate, ubiquitous eruptions could play a role in the occurrence of larger scale CMEs reported in the previous decades. Here however, we will investigate the possible role played by large scale CMEs in the occurrence of small scale eruptions. To this end, we first report on the detection of secondary eruptions induced by a CME expansion (Sect. 2), second we show that these eruptions can be simulated by allowing magnetic reconnection in the ambient corona where the CME expands (Sect. 3); our results are then summarized in Sect. 4.

\section{Observations}

The coronal white light configuration on 10 December 2005 is shown in Fig. 1 (panel a): in particular SOHO/LASCO 

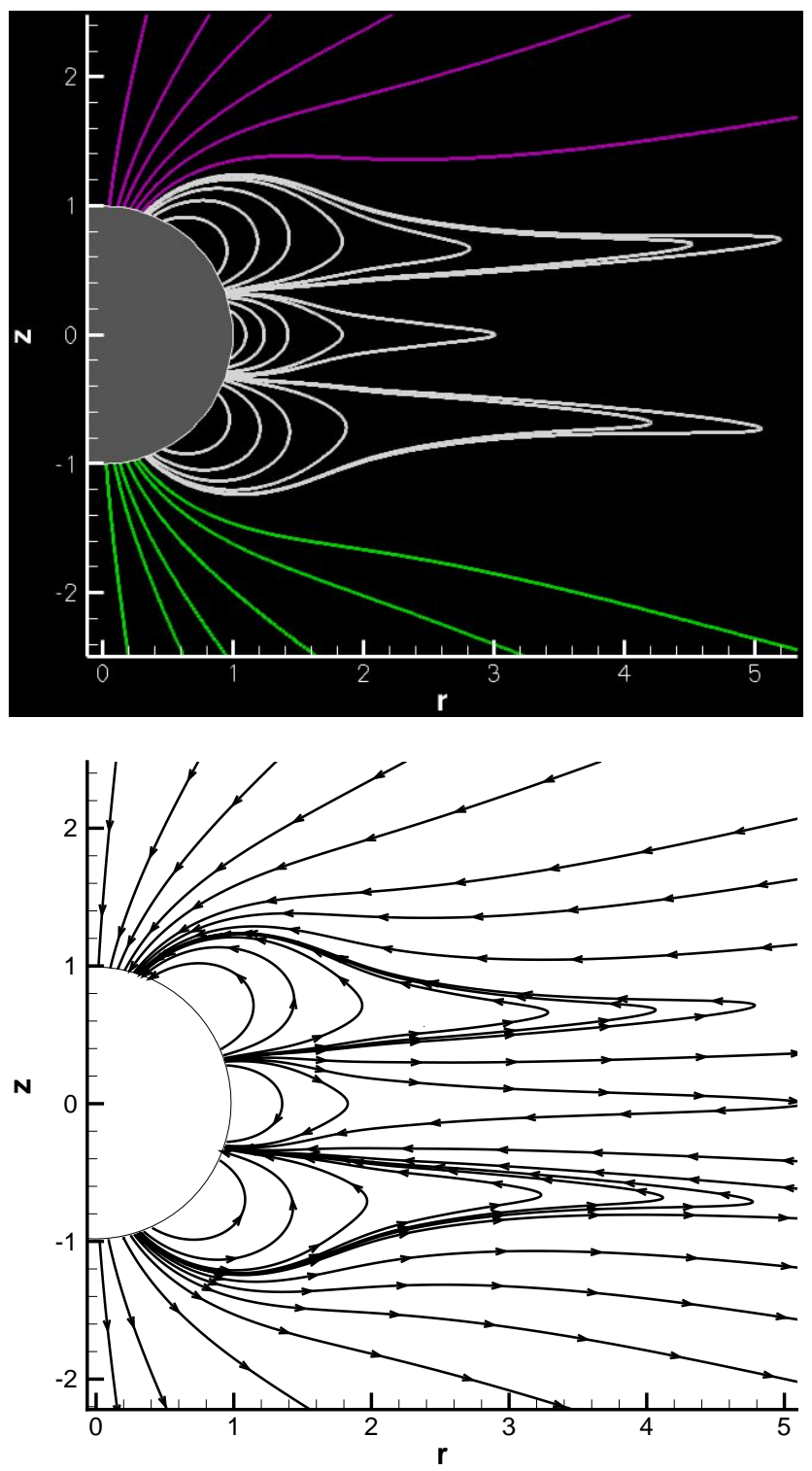

Fig. 3. Top: The initial coronal configuration used in our simulations obtained by the potential superpositoin of a dipolar and a quadrupolar configuration. The purple and green colored field lines represent regions of open field lines and fast flow while the three streamers are colored in white. Bottom: The initial coronal configuration showing the orientation of the magnetic field lines.

C2 images show two streamers centered at an approximate latitude of $39^{\circ} \mathrm{NW}$ and $40^{\circ} \mathrm{SW}$ and a complex of smaller radial structures in between (Fig. 1). Starting at 19:00 UT, a CME appears at the West limb in the LASCO/C2 field of view. This CME propagated in between the two streamers, with its flanks close to the streamer axes. Starting from about 03:00 UT on 11 December, the northwest streamer appears to pinch off and the propagation of a concave Y-shaped feature along the streamer axis is observed (Fig. 1, panels bc). In the following hours, after a small secondary ejection that occurred around 12:00UT (Fig. 1, panel d), a similar process occurs in the southwest streamer that clearly shows the formation of an X-point in between two radial features approaching each other. The X-points stretches in two Yshaped features, one propagating outward along the streamer and the other rooted on the Sun, while a current-sheet-like pattern develops in between (Fig. 1, panel e).

Figure 4 shows the system's evolution over time of the relative density in response to the applied shearing motions. The colour code indicates the relative density, i.e. $\rho_{r}(t) \simeq[\rho(t)-\rho(0)] / \rho(0)$, while the white lines represent the magnetic field lines.

A possible interpretation for the series of events described above has been provided by Bemporad et al. (2008); in this work they proposed that the observed secondary eruptions could result from magnetic reconnection occurring in the lower corona (above or below the altitude of the LASCO/C2 occulter) along the neutral streamer current sheets or between the CME flanks and the streamer boundaries; in our interpretation these events were induced by the CME expansion. In particular, one of the above reconnection events has been directly observed by the SOHO/UVCS instrument (the "Ultra Violet Coronagraph Spectrometer"): the UVCS spectroscopic data have been used to estimate the coronal plasma physical parameters before and after the reconnection and in particular (for details, see Bemporad et al., 2008). In the following we will demonstrate that the proposed interpretation can be reproduced by MHD simulations.

To this end, a better knowledge of the pre-CME coronal magnetic field is needed. Figure 2 shows the photospheric field map (observed by SOHO/MDI) along with the extrapolated coronal magnetic field. Strong bipolar photospheric fields (up to $\sim 10^{3} \mathrm{G}$ ) around the equator at the West limb are associated with a complex of Active Regions that, on 11 December 2005, was approximately crossing the limb. At higher North and South latitudes the "S"-shaped magnetic neutral line is mainly parallel to the equator. Hence, along the West limb we find a stronc concentrated bipole around the equator and two more dispersed and weaker bipoles ar larger latitudes ("esa-polar configuration") Corresponding coronal magnetic fields were computed starting from photospheric fields using a PFSS (Potential Field Source Surface) approximation. Within the potential field approximation dynamic phenomena such as solar eruptions cannot be reproduced, but it has been demonstrated that this approximation can provide a good tool to estimate the magnetic field configuration in coronal streamers. In particular, the extrapolated field shows two middle latitude groups of extended closed fieldlines (in very good agreement with the positions of the two observed coronal streamers) and a smaller equatorial group associated with the CME source region. In the following Section we will demonstrate that, using as a starting point an initial magnetic configuration of coronal fields that mimics the observed configuration it is possible to reproduce the secondary eruptions observed in white light coronagraphs and induced by the CME expansion. 

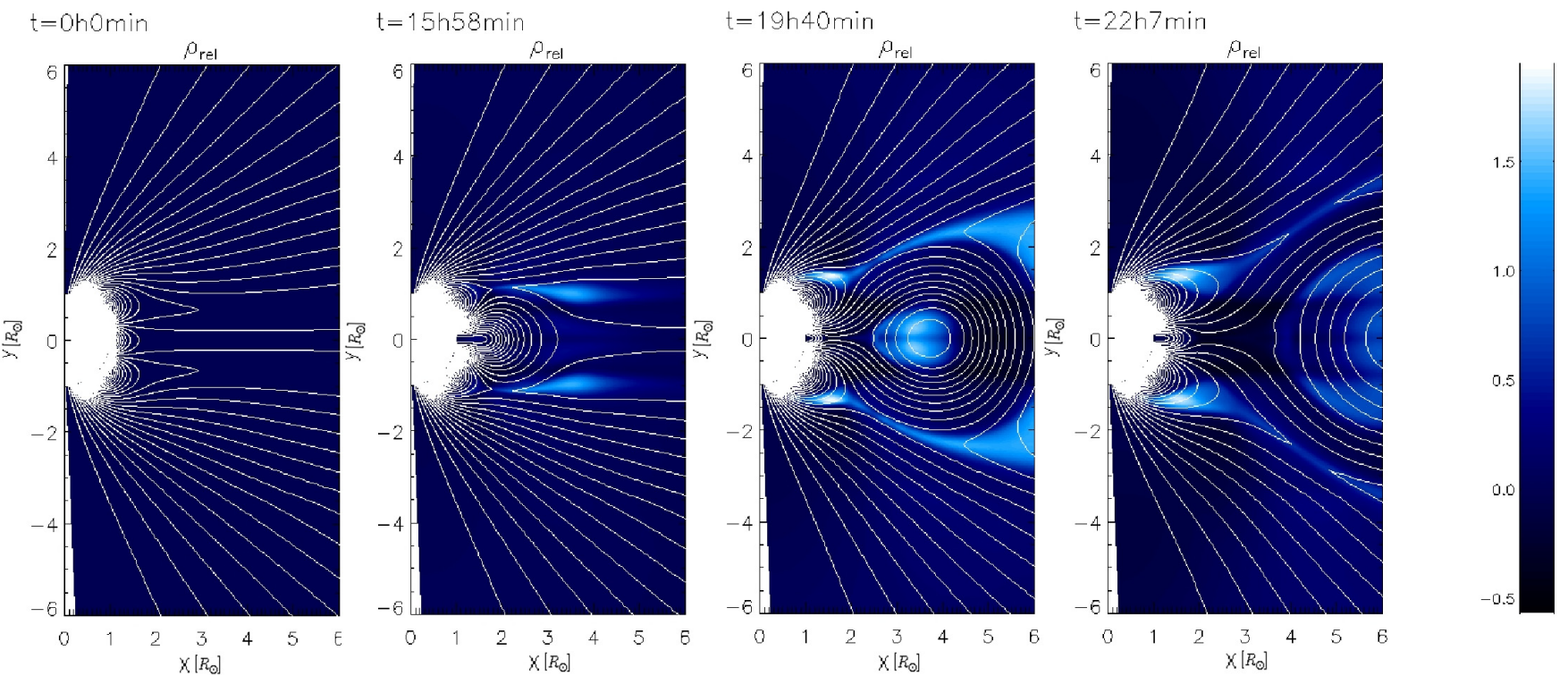

Fig. 4. Snapshots showing the relative mass density and magnetic field lines at different times during our simulation. The left panel shows the initial configuration. The second panel shows how the relative density has evolved after $t=15 \mathrm{~h} 58 \mathrm{~min}$. The third panel shows how the relative density has evolved after $t=19 \mathrm{~h} 40 \mathrm{~min}$ and clearly shows how the expanding CME is pinching the northern and southern streamers. The fourth panel shows the evolution after $t=22 \mathrm{~h} 7 \mathrm{~min}$ when we can clearly see how induced lateral reconnection has detached the top parts of the two streamers.
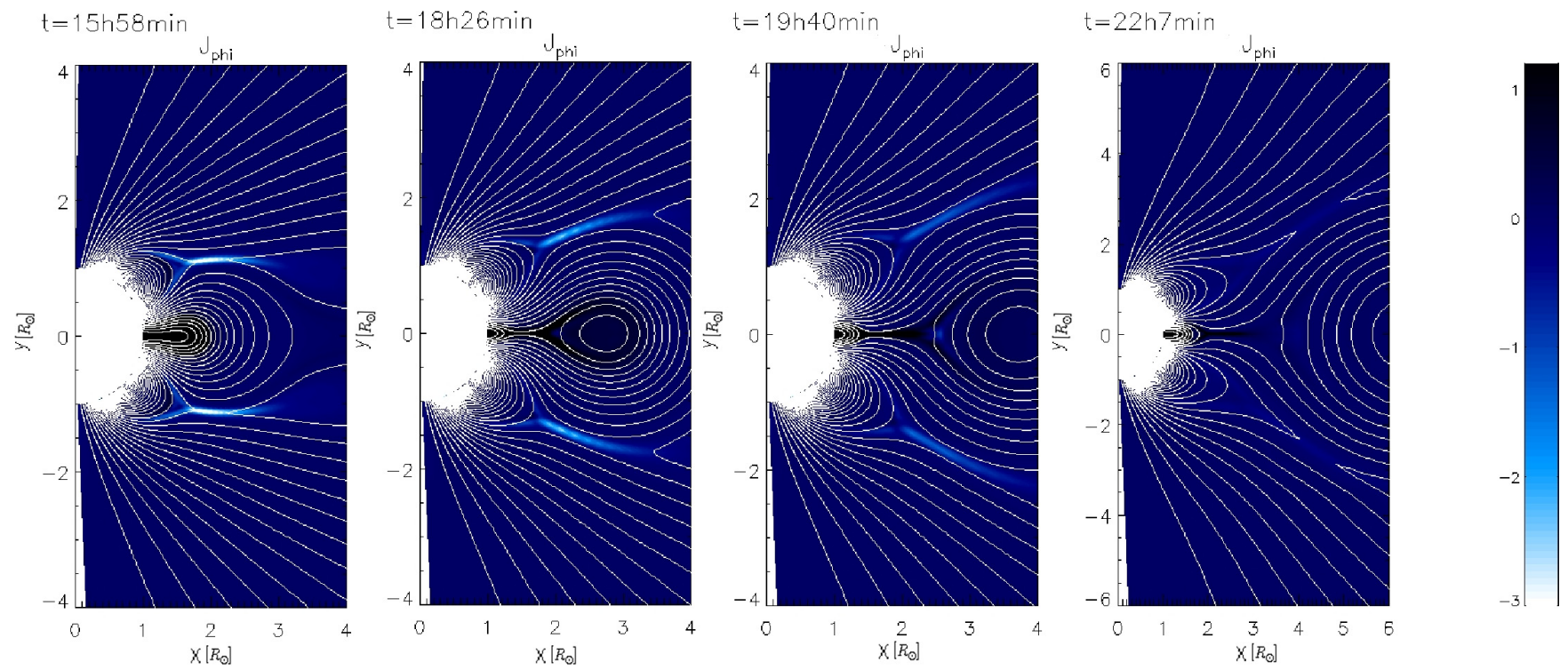

Fig. 5. Snapshots showing the current density and magnetic field lines at different times during our simulation. The left panel shows the current density at $t=15 \mathrm{~h} 58 \mathrm{~min}$. The second panel shows the reconnection at $t=18 \mathrm{~h} 26 \mathrm{~min}$ when the expanding central arcade pushes the northern and southern streamers and induces reconnection. The third panel shows the current density at $t=19 \mathrm{~h} 40 \mathrm{~min}$ while the fourth panel shows the current density at $t=22 \mathrm{~h} 7 \mathrm{~min}$. Here the tops of streamers have already detached.

\section{Simulations}

The solar corona, the initial arcades and the wind are modeled in the framework of ideal magnetohydrodynamics (MHD). The MHD equations are solved numerically in spherical geometry and assuming axisymmetry (2.5D). The grid contains $484 \times 205$ cells. The initial condition is a bimodal solar wind with fast flow at the poles and slow flow around the equatorial region. The coronal magnetic field is the potential superposition of a dipolar and a quadrupolar 
configuration resulting in the triple streamer structure shown in Fig. 3. Notice that the simulated initial configuration is very similar to the configuration extrapolated from the observed photospheric fields. The system is then driven by shearing motions at the footpoints of the central structure.

An extra upward magnetic pressure force is built up due to the extra azimuthal magnetic field, $B_{\varphi}$, generated at the solar base in response to the applied shearing motions, eventually causing the central structure to expand outwards. This outward expansion leads to the formation of a flux rope. Figure 4, first panel, shows the initial steady state coronal configuration. The plasmoid has expanded outward significantly after $t=15 \mathrm{~h} 58 \mathrm{~min}$, Fig. 4 second panel. The northwest and southwest streamers are pushed northward and southward respectively by the expanding field lines and the plasma in these streamers is compressed as is demonstrated by the increase in relative density in these regions. Figure 4 third panel, shows how the central structure (or streamer) has continued to expand outward. The density at the centre has also increased significantly. As it rises outward, the plasma within the central streamer is accelerated into the solar wind, stretching it even further. Eventually a current sheet is created with which the lower-lying loops can reconnect. Meanwhile the nothern and southern streamers have continued to be pushed up- and downward compressing the plasma inside even further. Finally, the flux rope is ejected from the solar surface. Figure 4, fourth panel, shows how the CME has expanded outward even further and consists of a bright leading edge, a cavity and a dense core embedded in this cavity. It's further expansion has also induced even more intense reconnection with the two streamers resulting in the detachment of the top of both streamers (at $\approx 4.5 R_{\odot}$ ). Because of the axisymmetric nature of our simulations, these detachments occur simultaneously for both the northern and the southern streamer. They propagate outwards as concave Y-shaped features. Towards the end of our shear profile, the streamer reforms and we return to our initial triple streamer configuration.

Figure 5 shows the evolution of the current density over time. The current density is used to indicate where reconnection in our system takes place. In Fig. 5 first panel shows how the reconnection takes place between the flank of the expanding central structure and the adjacent streamers. The reconnection site is pushed north- and southward as the central streamer continues to expand, as shown in Fig. 5 second panel. The reconnection site continues to move to a higher latitude in Fig. 5 third panel and Fig. 5 fourth panel until the central structure is detached from the solar surface and the tops of the adjacent streamers disconnected by the induced reconnection.

The current symmetrical setup does not allow the reproduction of the observed secondary eruptions. The observations report two streamers centered at an approximate latitude of $39^{\circ} \mathrm{NW}$ and $40^{\circ} \mathrm{SW}$ (18:00 UT) and a complex of smaller radial structures in between. The two streamers in

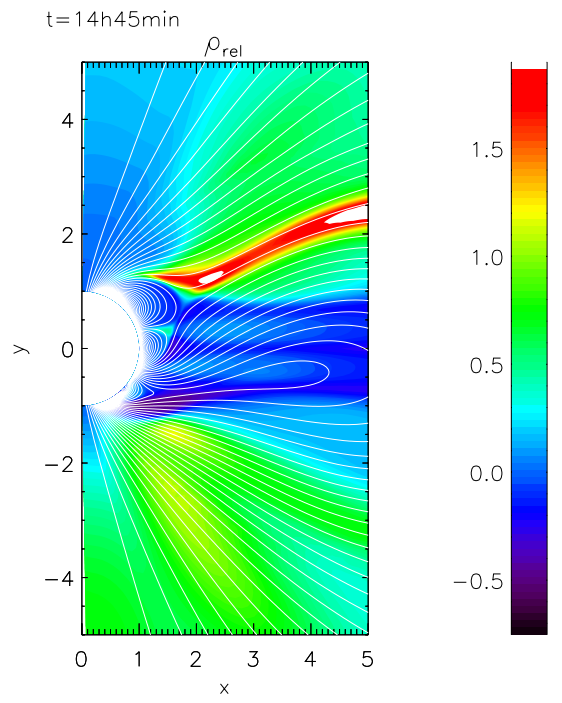

Fig. 6. Snapshot of the relative density and magnetic field lines showing the secondary eruption in the wake of the disconnected streamer top (marked in white).

our simulations are centered at a latitude of $35^{\circ} \mathrm{NW}$ and $35^{\circ} \mathrm{SW}$. The complex of smaller radial structures is represented by a single streamer centered around the equator. We will only be able to reproduce the secondary eruptions if we place a more complex structure in between the two streamers or if we move away from a perfectly symmetric evolution of the system. The latter can be done by simply shearing either the NW or SW streamer. Introducing extra azimuthal magnetic field, $B_{\varphi}$, at the base of the SW streamer causes it to swell up. The central and NW streamers are pushed northward by the expanding flux rope. Reconnection causes the tops of both streamers to eventually disconnect. However, reconnection between the CME flank and the NW and central streamer causes a small secondary eruption that can be seen in Fig. 6. The secondary eruption follows the disconnection of the streamer tops. Further simulations and more detailed analysis will be reported elsewhere.

\section{Conclusions}

White light observations reported here demonstrate that, during a CME expansion, many small scale secondary ejections of plasma may occur in the surrounding corona during and after the main eruption. In our interpretation these secondary eruptions originate from reconnection events induced by the expanding CME in the surrounding corona along the streamer current sheets and between the CME flanks and the streamer boundaries (see also Bemporad et al., 2008). Simulations reported here have shown that this interpretation is correct because secondary side ejections induced by an expanding CME can be, at least qualitatively, reproduced. Our simulations started from an initial magnetic 
field configuration that mimics the real field configuration as extrapolated in the corona from photospheric fields using a PFSS approximation. Our initial axisymmetric simulations were unable to reproduce the observed secondary eruptions but were able to reproduce the streamer detachments. However, when the bottom streamer was stressed instead of the central structure, a secondary eruption was observed. Reproducing the observed secondary eruptions more accurately would require us to start from a non-symmetric initial configuration, which we plan to do in the near future. There is a clear indication however that CMEs could not only be associated to a single location of reconnection in the corona, but to many reconnections occurring at different times and locations around the flux rope. The exact effect of these lateral reconnections is currently being studied in more detail; in any case first results reported here show that future CME models will have to take this "lateral reconnection effect" into account.

Acknowledgements. The authors thank ISSI (International Space Science Institute, Bern) for the hospitality provided to the members of the team on the Role of Current Sheets in the Solar Eruptive Phenomena, where many ideas presented in this work have been discussed. A.B. acknowledges support from ASI/INAF I/035/05/0 contract. SOHO is a Mission of international cooperation between ESA and NASA. A.S. acknowledges support from the projects GOA/2009-009 (K.U. Leuven), G.0304.07 (FWO-Vlaanderen) and C 90205 (ESA Prodex 9). The numerical results were obtained on the HPC cluster VIC of the K.U. Leuven.

Topical Editor R. Forsyth thanks B. Vrsnak and another anonymous referee for their help in evaluating this paper.

\section{References}

Amari, T., Luciani, J. F., Mikić, Z., and Linker, J.: A twisted flux rope model for coronal mass ejections and two-ribbon flares, ApJ, 529, L49-L52, 2000.

Amari, T., Luciani, J. F., Aly, J. J., et al.: Coronal mass ejection: initiation, magnetic helicity, and flux ropes. I. Boundary motiondriven evolution, ApJ, 585, 1073-1086, 2003.

Amari, T., Aly, J. J., Mikić, Z., and Linker, J.: Coronal Mass Ejection Initiation and Complex Topology Configurations in the Flux Cancellation and Breakout Models, ApJ, 671, L189-L192, 2007.

Antiochos, S. K., DeVore C. R., and Klimchuk, J. A.: A model for solar coronal mass ejections, ApJ, 510, 485-493, 1999.

Attrill, G. D. R., Harra, L. K., van Driel-Gesztelyi, L., and Démoulin, P.: Coronal "wave": Magnetic Footprint of a Coronal Mass Ejection?, ApJ, 656, L101-L104, 2007.

Bárta, M., Vršnak, B., and Karlický, M.: Dynamics of plasmoids formed by the current sheet tearing, A\&A, 477, 649-655, 2008.

Bemporad, A., Poletto, G., Landini, F., and Romoli, M.: Magnetic reconnection processes induced by a CME expansion, Ann. Geophys., 26, 3017-3024, 2008,

http://www.ann-geophys.net/26/3017/2008/.

Bemporad, A., Sterling, A. C., Moore, R. L., and Poletto, G.: A New Variety of Coronal Mass Ejection: Streamer Puffs from Compact Ejective Flares, ApJ, 635, L189-L192, 2005.
Bravo, S., Aquilar, E., Blanco-Cano, X., et al.: Coronal Magnetic structures associated with interplanetary clouds, Solar Phys., 188, 163-168, 1999.

Chen, Y., Li, X., Song, H. Q., et al.: Intrinsic Instability of Coronal Streamers, ApJ, 691, 1936-1942, 2009.

Chen, P. and Shibata, K.: An emerging flux trigger mechanism for coronal mass ejections, ApJ, 545, 524-531, 2000.

Cirtain, J. W., Golub, L., Lunduist, L., et al.: Evidence for Alfven Waves in Solar X-Ray Jets, Science, 318, 1580, doi:10.1126/science.1147050, 2007.

Démoulin, P., Klein, K.-L., Goff, C. P., et al.: Decametric N Burst: A consequence of the Interaction of Two Coronal Mass Ejections, Solar Phys., 240, 301-313, 2007.

Feynman, J. and Martin, S. F.: The initiation of coronal mass ejections by newly emerging magnetic flux, J. Geophys. Res., 100, 3355-3367, 1995.

Forbes, T. G., Linker, J. A., Chen, J., et al.: CME Theory and Models, Space Sci. Rev., 123, 251-302, 2006.

Gilbert, H. R., Serex, E. C., Holzer, T. E., et al.: Narrow Coronal Mass Ejections, ApJ, 550, 1093-1101, 2001.

Goff, C. P., van Driel-Gesztelyi, L., Démoulin, P., et al.: A Multiple Flare Scenario where the Classic Long-Duration Flare Was Not the Source of a CME, Solar Phys., 240, 283-299, 2007.

Gopalswamy, N.: Recent advances in the long-wavelength radio physics of the Sun, P\&SS, 52, 1399-1413, 2004.

Innes, D. E., Genetelli, A., Attie, R., and Potts, H. E.: Quiet Sun mini-coronal mass ejections activated by supergranural flows, A\&A, 495, 319-323, 2009.

Klimchuk, J. A.: Geophys. Monograph Series, Vol. 125, Space Weather, edited by: Song, P., Singer, H. J., and Siscoe, G. L., AGU, 143, 2001.

Ko, Y.-K., Raymond, J. C., Lin, J., et al.: Dynamical and Physical Properties of a Post-Coronal Mass Ejection Current Sheet, ApJ, 594, 1068-1084, 2003.

Low, B. C.: Coronal mass ejections, magnetic flux ropes, and solar magnetism, J. Geophys. Res., 106, 25141-25164, 2001.

Lynch, B. J., Antiochos, S. K., DeVore, C. R., et al.: Topological Evolution of a Fast Magnetic Breakout CME in Three Dimensions, ApJ, 683, 1192-1206, 2008.

Mandrini, C. H., Nakwacki, M. S., Attrill, G., et al.: Are CMERelated Dimmings Always a Simple Signature of Interplanetary Magnetic Cloud Footpoints?, Solar Phys., 244, 25-43, 2007.

Martin, S. F.: Signs of Helicity in solar prominences and related features, Adv. Space Res., 32, 1883-1893, 2003.

Mikic, Z. and Linker, J. A.: Disruption of coronal magnetic field arcades, ApJ, 430, 898-912, 1994.

Mikic, Z. and Lee, M. A.: An Introduction to Theory and Models of CMEs, Shocks, and Solar Energetic Particles, Space Sci. Rev., 123, 57-80, 2006.

Moore, R. L. and Sterling, A. C.: The Coronal-dimming Footprint of a Streamer-Puff Coronal Mass Ejection: Confirmation of the Magnetic-Arch-Blowout Scenario, ApJ, 661, 543-550, 2007.

Nisticò, G., Bothmer, V., Patsourakos, S., and Zimbardo, G.: Observational features of equatorial coronal hole jets, Ann. Geophys., in review, 2009.

Roussev, I. I. and Sokolov, I. V.: in: Solar Eruptions and Energetic Particles, Geophys. Monograph Series, Vol. 165,, edited by: Gopalswamy, N., Mewaldt, R., and Torsti, J., AGU, 89-102, 2006. 
Rust, D. M. and Kumar, A.: Evidence for Helically Kinked Magnetic Flux Ropes in Solar Eruptions, ApJ, 464, L199, doi:10.1086/310118, 1996.

Sheeley Jr., N. R. and Wang, Y.-M.: In/Out Pairs and the Detachment of Coronal Streamers, ApJ, 655, 1142-1156, 2007.

Shibata, K., Nakamura, T., Matsumoto, T., et al.: Chromospheric Anemone Jets as Evidence of Ubiquitous Reconnection, Science, 318, 1591, doi:10.1126/science.1146708, 2007.

Sterling, A. C. and Hudson, H. S.: YOHKOH SXT Observations of X-Ray "Dimming" Associated with a Halo Coronal Mass Ejection, ApJ, 491, L55+, 1997.

van Ballegooijen, A. A. and Martens, P. C. H.: Formation and eruption of solar prominences, ApJ, 343, 971-984, 1989. van Driel-Gesztelyi, L., Attrill, G. D. R., Démoulin, P., Mandrini, C. H., and Harra, L. K.: Why are CMEs large-scale coronal events: nature or nurture?, Ann. Geophys., 26, 3077-3088, 2008, http://www.ann-geophys.net/26/3077/2008/.

Vourlidas, A., Buzasi, D., Howard, R. A., and Esfandiari, E.: in: "Solar Variability: From Core to Outer Frontiers", 506, 91-94, 2002.

Wang, Y.-M. and Sheeley Jr., N. R.: Observations of Flux Rope Formation in the Outer Corona, ApJ, 650, 1172-1183, 2006.

Zuccarello, F. P., Soenen, A., Poedts, S., et al.: Initiation of Coronal Mass Ejections by Magnetic Flux Emergence in the Framework of the Breakout Model, ApJ, 689, L157-L160, 2008. 